\title{
Optimizing glass-ceramic bonding incorporating new silane technology in an experimental universal adhesive formulation
}

Chenmin Yao ${ }^{a, b}$, Mohammed H. Ahmed ${ }^{a, c}$, Lauren De Grave ${ }^{d}$, Kumiko Yoshiharae,f, Ben Mercelis ${ }^{a}$, Yohei Okazaki ${ }^{a, g}$, Kirsten L. Van Landuyt ${ }^{a}$, Cui Huang ${ }^{b}$, Bart Van Meerbeek ${ }^{{ }^{*}}$

${ }^{a} \mathrm{KU}$ Leuven (University of Leuven), Department of Oral Health Sciences, BIOMAT \& UZ Leuven (University Hospitals Leuven), Dentistry, 3000 Leuven, Belgium

${ }^{b}$ Wuhan University, the State Key Laboratory Breeding Base of Basic Science of Stomatology (HubeiMOST) \& Key Laboratory of Oral Biomedicine Ministry of Education (KLOBM), School \& Hospital of Stomatology, 430079 Wuhan, China

'Tanta University, Faculty of Dentistry, Department of Dental Biomaterials, 31511 Tanta, Egypt

${ }^{d} \mathrm{KU}$ Leuven (University of Leuven), Department of Chemistry, Polymer Chemistry and Materials, 3000 Leuven, Belgium

eNational Institute of Advanced Industrial Science and Technology (AIST), Health and Medical Research Institute, 761-0395 Takamatsu, Japan

fOkayama University, Graduate School of Medicine, Dentistry and Pharmaceutical Sciences, Department of Pathology \& Experimental Medicine, 700-8556 Okayama, Japan

${ }^{g}$ Hiroshima University, Department of Advanced Prosthodontics, 734-8553 Hiroshima, Japan

Corresponding author: Prof. Dr. Bart Van Meerbeek, KU Leuven (University of Leuven), Department of Oral Health Sciences, BIOMAT, Kapucijnenvoer 7, blok a - box 7001, BE-3000 Leuven, Belgium. TEL: +32-16-337587, FAX: +32-16-332752, bart.vanmeerbeek@kuleuven.be

Chenmin Yao, Mohammed H. Ahmed, Lauren De Grave, Kumiko Yoshihara, Ben Mercelis, Yohei Okazaki, Kirsten L. Van Landuyt, Cui Huang, Bart Van Meerbeek. Optimizing glass-ceramic bonding incorporating new silane technology in an experimental universal adhesive formulation. Dental Materials. 


\section{ABSTRACT}

Objectives. Incorporating silane-coupling agent into universal adhesives (UAs) to simplify adhesive luting of glass-ceramic restorations appeared ineffective due to silane's instability in an acidic aqueous solution. This study aimed to evaluate new silane technology added to an experimental UA to be bonded to glass ceramics without separate prior silanization.

Methods. Combined silane technology, consisting of 3-(aminopropyl)triethoxysilane (APTES) and $\gamma$ methacryloxypropyltriethoxysilane (YMPTES), was incorporated into an experimental UA formulation, being referred to as ADH-XTE (3M Oral Care). Immediate and aged shear bond strength (SBS) of ADH$\underline{\mathrm{XTE} \text { onto }}$ as-milled ('AM'), tribochemical silica-coated ('TSC'), HF-etched ('HF'), and mirror-polished ('MP') glass-ceramic CAD/CAM blocks (IPS e.max CAD) with/without separate silanization was measured ( $n=10 /$ group). The control adhesives included Scotchbond Universal ('SBU') and Scotchbond $1 \mathrm{XT}($ (SB1-XT'). The glass-ceramic surface topography and the fractography of the SBS-debonded specimens were observed by SEM.

Results. Without separate prior silanization, the experimental UA ADH-XTE, containing combined APTES/YMPTES silane technology, significantly outperformed the glass-ceramic bonding efficiency of its silane-containing SBU precursor, while it performed equally effective as SBU applied with prior silanization. Upon aging, significant reduction in SBS was recorded when ADH-XTE was bonded to TSC glass-ceramic surfaces $(p<0.05)$, while not to HF ones. Notably, the lowest SBS was obtained when the $\underline{\text { UAs were bonded to AM and MP glass-ceramic surfaces, in particular when applied without separate }}$ prior silanization $(p<0.05)$.

Significance. The glass-ceramic bonding capacity of the new combined APTES/YMPTES silanecontaining UA ADH-XTE surpassed that of its SBU precursor. HF etching remains needed to durably bond to glass-ceramics.

Keywords:

Adhesive luting; Silanization; 3-(aminopropyl)triethoxysilane; APTES; үMPTES; үMPTS 


\section{Introduction}

The clinically best performing protocol to adhesively lute glass-ceramic restorations involves etching using hydrofluoric acid (HF) to enable surface micro-retention for the composite cement, followed by silanization to achieve additional chemical binding for bond durability $[1,2]$. The functional monomer $\gamma$-methacryloxypropyltrimethoxysilane ( $\gamma$ MPTS) is the most commonly used silane in dentistry $[3,4]$. Bifunctional silane forms a siloxane network at the etched glass-ceramic surface by condensation and copolymerizes with methacrylate groups of the optionally applied adhesive resin (without being separately light-cured) and/or the luting composite [5, 6]. Various silane-containing (i.e., YMPTS) universal adhesives (UAs) have emerged onto the dental market to simplify and shorten adhesive luting procedures, claiming that additional silanization with a separate ceramic primer is no longer needed [7]. However, mechanistic research showed that YMPTS is not stable in an acidic aqueous solution like a UA, by which application of a separate silane primer is still recommended $[3,8,9]$.

The hydrolysis rate of silane depends not only on the solution's pH and silane concentration, but also on the molecular structure of the actual silane bifunctional monomer [4]. Whether other silanes are more stable in aqueous acidic UAs, is not yet been sufficiently known. APTES, representing 3(aminopropyl)triethoxysilane, is a potential candidate compound to be added to an UA. It has $\underline{\text { frequently been used in biotechnology and contains an amino-terminal group and functional alkoxy }}$ groups [10-12]. Upon hydrolysis of the alkoxy groups, APTES can chemically react with hydroxyl groups at the ceramic surface through its silanol. Additionally, $\gamma$-methacryloxypropyltriethoxysilane ( which is an YMPTS analogue, can be used to react via radical polymerization with the functional $(C=C)$ vinyl groups of the monomers within the adhesive resin. Upon irradiation with light, the photo-initiator within the adhesive dissociates first into free radicals [13]. Next, the silane monomer co-polymerizes with methacrylates of the adhesive resin, forming new C-C bonds [14]. Based on the abovementioned, a new experimental UA ADH-XTE (3M Oral Care, Seefeld, Germany) has been developed, containing both APTES and the YMPTES. The chemical structures of APTES, YMPTES and YMPTS are shown in Fig. 1. The combined APTES/YMPTES silane technology is expected to enhance the bonding performance to glass ceramics. However, research questions on the ADH-XTE bonding potential when applied without a separate ceramic primer and if it hereby overcomes the prior reported problem of silane instability in UAs need to be figured out. 
This study therefore aimed to evaluate the bonding potential of ADH-XTE to four diversely prepared glass-ceramic surfaces. The null hypothesis tested was that the combined APTES/YMPTES silanecontaining UA ADH-XTE did not bond more effectively to glass ceramics than its YMPTS silanecontaining precursor UA Scotchbond Universal (3M Oral Care), this (1) irrespective of glass-ceramic surface pre-treatment and (2) with/without separate application of a ceramic primer.

\section{Materials and methods}

\subsection{Preparation of glass-ceramic surfaces}

Lithium-disilicate glass-ceramic blocks (IPS e.max CAD, Ivoclar Vivadent, Schaan, Liechtenstein) were sectioned into 12 pieces $(8 \times 7 \times 4 \mathrm{~mm})$ using a low-speed cutting device (Micracut 151, Metkon, Bursa, Turkey) combined with an automatic precision cutting machine (Accutom-50, Struers, Ballerup, Denmark). Glass-ceramic specimens ( $n=412)$ were sintered (P300, Ivoclar Vivadent) according to the manufacturer instructions, prior to being embedded in auto-cure acrylic resin and ground with FEPA P320 silicon carbide (SiC) paper (Buehler, Lake Bluff, IL, USA) using a polishing machine (Beta grinder polisher, Buehler) for $1 \mathrm{~min}$ to produce a CAD/CAM-milled representative surface. The as-milled ('AM') glass-ceramic surfaces directly received the adhesive luting procedure (first specimen set) or were further treated following three additional experimental conditions $(n=103)$.

Specimens in the second set were tribochemically silica coated ('TSC') using an intraoral microblasting device (CoJet Prep, 3M Oral Care) filled with 30- $\mu \mathrm{m}$ silica-coated aluminum oxide powder (CoJet Sand, 3M Oral Care) and applied with a work distance of $5 \mathrm{~mm}$ at an air pressure of $2.8 \mathrm{bar}$ (40 psi) for $15 \mathrm{~s}$. The surfaces were next cleaned by a stream of dry, oil-free air to remove any residual powder. Specimens in the third set ('HF') were etched with $2.5-<7 \%$ HF (IPS Ceramic Etching Gel, Ivoclar Vivadent; $\mathrm{pH}=2$ at $20^{\circ} \mathrm{C}$ according to the manufacturer's safety data sheet) for $20 \mathrm{~s}$, followed by thoroughly rinsing under running tap water for $60 \mathrm{~s}$. All specimens were post-etched with phosphoric acid (3M Universal Etchant, 3M Oral Care) for $20 \mathrm{~s}$ to remove any residue and finally air dried. Specimens of the final fourth set were sequentially ground with P1200 and P4000 SiC papers (Struers) and mirror polished ('MP') with 3.0-, 1.0- and 0.5- $\mu \mathrm{m}$ diamond suspensions (Kemet International, Maidenstone, UK) dispensed on cloth paper (MicroCloth, PSA, Buehler) using the Beta grinder polisher 
(Buehler). The specimens were rinsed with distilled water for $1 \mathrm{~min}$ and submerged in absolute ethanol for 10 s after each polishing step to fully remove polishing debris, prior to being dried with an ordinary hair dryer. Polishing was continued towards mirror gloss without surface scratches, as evaluated by light microscopy (Stemi 2000-CS, Zeiss, Oberkochen, Germany).

\subsection{SEM surface characterization}

The AM, TSC, HF and MP specimens ( $n=3$ ) were coated with gold using a gold-sputter coater (JFC-1300, Jeol, Tokyo, Japan) for $40 \mathrm{~s}$. The surface structure was characterized by SEM (JSM-6610LV, Jeol) at $5 \mathrm{kV}$ with photomicrographs taken at $1000 \times$ and $10000 \times$ original magnification.

\subsection{Shear bond strength (SBS)}

Four hundred glass-ceramic plates were assigned to 40 experimental groups ( $n=10$ per group): 4 substrate pretreatments (i.e., AM, TSC, HF and MP) $\times 5$ silanization/adhesive protocols (see below) $\times 2$ aging periods (1-week and 3-month water storage). The five silanization/adhesive protocols involved (a) sole application of the experimental combined APTES/YMPTES silane-containing UA ADH-XTE (3M

Oral Care) without separate ceramic primer, (b) separate silanization using RelyX Ceramic Primer ('RCP', 3M Oral Care; composition: YMPTS, ethanol and water) followed by ADH-XTE, (c) sole application of the YMPTS silane-containing UA Scotchbond Universal (SBU; 3M Oral Care), (d) separate RCP silanization followed by SBU, and (e) separate RCP silanization followed by the two-step etch-and-rinse adhesive (2-E\&Ra) Scotchbond 1 XT ('SB1-XT'; 3M Oral Care) that does not contain silane and served as control. The compositions of dental adhesives investigated in this study are detailed in the Table 1.

All three adhesives were applied strictly following the manufacturer's instructions (Table 1), using Ultradent Bonding Clamps with Bonding Mold Inserts (Ultradent, South Jordan, UT, USA) to conduct a notched-edge SBS test according to the ISO 29022 standard. Restorative composite (Filtek Z250, 3M Oral Care) was applied in a 2-mm layer and light-cured using a polywave light-emitting diode (LED) curing light (Bluephase 20i, Ivoclar Vivadent) with an output of $1200 \mathrm{~mW} / \mathrm{cm}^{2}$ for $20 \mathrm{~s}$ when used in 'high mode'. Additionally, a thin and final 0.5-mm composite layer was added and light cured for $20 \mathrm{~s}$, so to ensure optimum curing. The light output was determined and confirmed regularly during the experiment using a Marc Resin Calibrator (BlueLight Analytics, Halifax, Canada). Upon light curing, all 
specimens were kept at $100 \%$ humidity for $1 \mathrm{~h}\left(37^{\circ} \mathrm{C}\right)$, prior to being immersed into prewarmed water $\left(37^{\circ} \mathrm{C}\right)$ for either 1 week $(1 \mathrm{w})$ or 3 months $(3 \mathrm{~m})$. The specimens were next mounted into a material tester (5848 Micro Tester, Instron, Norwood, MA, USA) using Ultradent Test Base Clamps combined with an Ultradent Crosshead Assembly, prior to being subjected to shear stress at a crosshead speed of $1 \mathrm{~mm} / \mathrm{min}$. When the specimens failed before actual testing, they were recorded as pre-test failures (ptfs).

\subsection{Failure-mode analysis and SEM of fractured surface}

All fractured specimens were analyzed under 40x magnification using light microscopy (Stemi 2000-CS, Zeiss) to determine the mode of failure, which was classified as either 'cohesive failure in ceramic', 'adhesive failure at the adhesive-ceramic interface', 'cohesive failure in adhesive', 'cohesive failure in composite' or 'mixed failure'. Representative fractured surfaces with a SBS close to the mean (2 specimens per experimental group), and specimens being recorded as ptf, were further examined by SEM at $5 \mathrm{kV}$ and an original magnification of $35 x$, this upon water cleaning, air-drying, and 40-s thinfilm gold-coating (JFC-1300, Jeol) of the specimens. Additionally, 2-3 spots at the central and peripheral area of the fractured surfaces were observed at a magnification of $2000 \times$ and $10000 \times$.

\subsection{Statistical analysis}

The SBS results were analyzed using multiple-way ANOVA (R3.1.0; R Foundation for Statistical Computing, Vienna, Austria) with specific contrasts and $p<0.05$ considered as being statistically significant. Data were modelled using generalized linear modelling of the four variables 'surface treatment' (AM, TSC, HF vs. MP), 'Ceramic Primer' (applied separately vs. not applied), 'adhesive' (ADHXTE vs. SBU) and 'aging' (1w vs. $3 \mathrm{~m}$ ). The reference consisted of separate silanization (RCP) followed by the silane-free 2-E\&Ra adhesive application of SB1-XT.

\section{Results}

\subsection{SEM surface characterization (Fig. 2)}

Glass-ceramic surface scratches, revealing additional surface irregularities at higher magnification, 
though with limited micro-retention potential, were observed at AM surfaces (Fig. 2a,b). A significantly rougher surface was exposed by TSC, which besides increased surface roughness should be attributed to silica-coated alumina particles deposited on the glass-ceramic surface, as can clearly be observed at higher magnification (Fig. 2c,d). A clean and clearly the most micro-retentive glass-ceramic surface, exhibiting exposed lithium-disilicate crystals at higher magnification, resulted from dissolution of surface glass phase by HF (Fig. 2e,f). MP resulted in an almost perfectly smooth surface without hardly any surface micro-retention available (Fig. 2g,h).

\subsection{SBS (Figs. $\underline{3}$ and 4 )}

The $1 \mathrm{w}$ and aged $3 \mathrm{~m}$ SBS data are graphically presented in box plots for the two UAs in Figs. 3 and 4, respectively, with the reference ' $\mathrm{RCP}+\mathrm{SB} 1-\mathrm{XT}$ ' SBS data presented as grey background boxes. The complete dataset is detailed in Table 2. Comparing bonding to the differently prepared glass-ceramic surfaces, the highest $1 \mathrm{w}$ and aged 3m SBSs were generally obtained when both UAs were bonded to HF surfaces (Figs. 3 and 4: grey vertical rectangles), because of which the key findings further reported primarily concern bonding to HF glass-ceramic surfaces. Comparing bonding of ADH-XTE to that of the $\mathrm{RCP}+\mathrm{SB1}-\mathrm{XT}$ reference (involving prior separate silanization) to HF surfaces (statistical significance indicated by a red X in Figs. 3 and 4), no significant difference in $1 \mathrm{w}$ and $3 \mathrm{~m}$ SBS was found, except for a significantly higher 1w SBS measured when a separate ceramic primer was applied prior to ADH-XTE $(p<0.05)$. Significantly lower $1 \mathrm{w}$ and 3m SBSs were however recorded for SBU when applied without prior separate silanization, as compared to those of the RCP+SB1-XT reference that included separate prior silanization $(p<0.05)$. When SBU was applied following separate silanization, no difference in $1 \mathrm{w}$ and $3 \mathrm{~m}$ SBS with those of the RCP+SB1-XT reference was recorded. Comparing bonding of the APTES/YMPTES silane-containing UA ADH-XTE to that of the YMPTS silane-containing SBU to HF-etched surfaces (statistical significance indicated by a black $\square$ in Figs. 3 and 4), a significantly higher $1 \mathrm{w}$ and $3 \mathrm{~m}$ SBS was recorded for ADH-XTE in all conditions except for the 1w SBS recorded with separate prior silanization. Moreover, the mean $1 \mathrm{w}$ and $3 \mathrm{~m}$ SBS of ADH-XTE applied without separate prior silanization to HF-etched surfaces corresponded to those of SBU applied with separate prior silanization (blue horizontal arrows). Evaluating the bond-promoting effect of prior separate

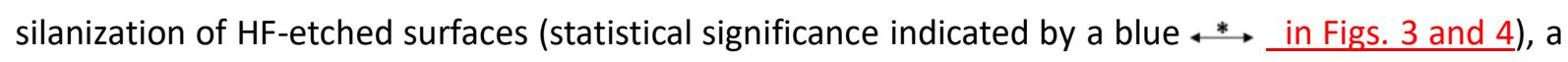


significantly higher SBS was measured for both UAs ADH-XTE and SBU at $1 \mathrm{w}$ and $3 \mathrm{~m}$ in all experimental conditions when a separate ceramic (silane) primer was applied prior to the UAs $(p<0.05)$. Evaluating the effect of aging ( $1 \mathrm{w}$ vs. $3 \mathrm{~m}$ SBS with statistical significance indicated by a red $\theta$ ), no significant decrease in SBS was recorded for ADH-XTE with/without separate prior silanization, while SBS of SBU without prior separate silanization statistically significantly decreased upon aging.

Overall, the APTES/YMPTES silane-containing UA ADH-XTE achieved better immediate bonding performance than its YMPTS silane-containing commercial precursor UA SBU $(p<0.05$; Fig. 3$)$, when both adhesives were applied without separate prior silanization to the pre-treated AM, TSC and HF glass-ceramic surfaces. However, no significant difference in immediate SBS was recorded for ADH-XTE and SBU when applied without separate prior silanization and bonded to the MP surfaces $(p>0.05)$. Likewise, the aged 3m SBS of ADH-XTE was significantly higher than that of SBU for all surface treatments with/without separate prior silanization $(p<0.05$; Fig. 4$)$, except solely when both adhesives were bonded to AM surfaces without separate prior silanization_and to TSC surfaces with separate prior silanization.

While both TSC and HF surface treatments morphologically produced rougher surfaces and potentially higher micro-retention potential with improved bonding receptiveness, SBS to HF glassceramic surfaces was generally superior over that to TSC glass-ceramic surfaces, with the latter bond less withstanding water-storage aging (Fig. 4). Overall, the lowest SBS was obtained when the UAs were bonded to AM and MP glass-ceramic surfaces, in particular when applied without separate prior silanization (Figs. 3 and 4). Interestingly, when the UAs were bonded to non-retentive MP glass-ceramic surfaces, the bond-promoting effect of separate prior silanization was very pronounced, with SBS approximating that recorded for the respective UAs when they were bonded to the most microretentive HF-etched surfaces without separate prior silanization.

\subsection{Failure-mode analysis and SEM of fractured surface}

The SBS failure-mode analysis data are graphically presented for all experimental groups in Fig. $\underline{5}$. Upon immediate and aged water storage, a more predominant 'cohesive failure in adhesive' mode was found for HF glass-ceramic surfaces (not for SBU applied without separate prior silanization) (Fig. 5). When ADH-XTE and SBU were applied without separate prior silanization and bonded to AM, TSC and MP 
glass-ceramic surfaces, the overall predominant failure mode was 'adhesive failure at the adhesiveceramic interface', which generally corresponded with the lower SBSs measured.

Representative SEM photomicrographs of fractured TSC and HF specimens at $1 \mathrm{w}$ and $3 \mathrm{~m}$ are presented in Fig. 6 . Regarding the immediate and aged fractured TSC specimens, the adhesive-ceramic interface failed predominantly adhesively, when ADH-XTE was applied without/with prior silanization (Fig. $6 \mathrm{Aa}, \mathrm{d}$ and $6 \mathrm{Ba}, \mathrm{d})$. High magnification at $10000 \times$ disclosed the exposed silica-coated ceramic surface (Fig. 6Ac,f and 6Bc,f). However, regarding the immediate and aged fractured HF-etched specimens, mainly cohesive failures in adhesive resin occurred, when ADH-XTE was applied without/with prior silanization (Fig. $6 \mathrm{Ag}, j$ and $6 \mathrm{Bg}, \mathrm{j}$ ). Upon aging, the adhesive layer was still complete and uniform for RCP ADH-XTE at a magnification of 2000x (Fig. 6Bk).

\section{Discussion}

In this study, we mainly investigated the bonding effectiveness of a novel experimental APTES/ $\mathrm{YMPTES}$ silane-containing UA (ADH-XTE) without/with prior silanization onto four differently prepared lithiumdisilicate glass-ceramic surfaces. Its commercial precursor UA (SBU), which solely contains YMPTS as silane functional monomer, and a conventional 2-E\&Ra, which does not contain silane but was applied with prior silanization (RCP+SB1-XT), were likewise investigated and served as controls. We previously studied the efficacy and stability of YMPTS incorporated within UAs with regard to their bonding performance to etchable ceramics without separate prior silanization $[3,15]$. These studies revealed a negative outcome, basically concluding that YMPTS is unstable in aqueous acidic solutions and separate silanization remains recommended with silane-containing UAs. In response, a new UA was developed that contained not only vMPTES (yMPTS analogue), having a polymerizable vinyl group, but also the silane functional monomer APTES. Although limited data on the application of APTES in dentistry is available, APTES has widely been used in biomedical engineering and applied chemistry $[10,12,16]$.

Overall, the bonding performance of the new APTES/YMPTES silane-containing UA ADH-XTE bonded to HF-etched (and TSC-prepared) glass ceramics and applied without prior silanization was similar to that of the control, which involved separate silanization using a dedicated ceramic primer (RCP) followed by the application of the non-silane-containing 2E\&Ra (SB1-XT). In addition, ADH-XTE'S 
bonding effectiveness to all four surface-pretreated glass ceramics surpassed that of the solely YMPTS silane-containing SBU when both UAs were applied without prior silanization. Nevertheless, ADH-XTE applied with separate prior silanization (RCP) still performed significantly better to all four surfacepretreated glass ceramics than when ADH-XTE was applied without RCP. According to these results, the null hypothesis tested in this study that the combined APTES/ $Y$ MPTES silane-containing UA ADH-XTE did not bond more effectively to glass ceramics than its YMPTS silane-containing precursor SBU, this irrespective of glass-ceramic surface pre-treatment and with/without separate application of a ceramic primer, has to be rejected.

Comparing the glass-ceramic surface pretreatments providing the highest surface micro-retention potential (HF and TSC, as opposed to AM and MP), HF etching substantially ameliorated the bonding receptiveness of glass ceramics and in particular improved the bond durability of UAs to glass ceramics, while TSC hardly did. Tribochemical silica coating (TSC), as well as passive alumina-particle air abrasion (not studied here), has gained attracted attention as alternative ceramic surface pretreatment because of HF's causticness. Although TSC chemically activates ceramic surfaces by depositing a freshly reactive silica-rich layer [4], the current data confirm that HF etching remains the most effective surface pretreatment for lithium-disilicate glass ceramics. Moreover, HF improved the bond-degradation resistance of adhesive-ceramic interfaces produced by both UAs. In particular, the bond strength of ADH-XTE solely slightly (2-4\%) decreased upon aging regardless of prior silanization or not. On the contrary, bond strength in all TSC experimental groups significantly deteriorated over time. Even with prior silanization, the aged SBS remained ca. $46 \%$ below that of the respective immediate SBS, by which TSC cannot be recommended as effective alternative for HF etching of glass ceramics. A plausible explanation for the low bond-strength resistance of TSC adhesive-ceramic interfaces against aging may be due to the superficial attachment of the silica layer to the glass-ceramic surface. Upon aging, absorbed water molecules may weaken the attached silica coating, accelerating hydrolysis and degradation of the siloxane film [17]. Alternatively, TSC may have induced surface micro-cracking, resulting in regional stress raisers [18]. Such interfacial cracking along with continuous water uptake is expected to speed up the bond-degradation process [19].

The higher bonding effectiveness and improved bond durability upon $3 \mathrm{~m}$ water-storage aging of the UAs tested to HF-etched glass ceramic corresponded to a predominant 'cohesive failure in adhesive' 
mode versus absence of 'adhesive failure at the adhesive-ceramic interface' mode for all adhesives tested, except for SBU when applied without prior silanization. The latter experimental group revealed a predominant 'adhesive failure at the adhesive-ceramic interface' mode, which should be explained in light of the ineffective/instable in-built YMPTS silane functional monomer, as mentioned before. Overall, 'adhesive failure at the adhesive-ceramic interface' was the predominant failure mode recorded for most experimental groups, except for the HF experimental groups (excluding SBU) as mentioned above, which is in full agreement with previous findings where adhesive interfacial failures were typically associated with low(er) bond strengths and high(er) ptf numbers [20]. In light of the latter ptfs, it is noteworthy that numerous ptfs, which is less common with SBS testing, occurred upon $3 m$ water-storage aging when ADH-XTE (8 out of 10 specimens) and SBU (9 out of 10 specimens) were applied without prior silanization onto AM-prepared glass-ceramic surfaces, and also for SBU applied without prior silanization onto MP-prepared glass ceramic (6 out of 10 specimens). This clearly indicates that the pure chemical bonding to glass ceramic, considering that both the AM- and MPprepared surfaces provided, respectively, minimal and no micro-retention, was insufficient to resist bond degradation irrespective with/without prior silanization.

Our results also confirmed previous research $[3,8,21]$ that separate prior silanization significantly promoted the bonding effectiveness of SBU, in this study to all four surface-pretreated glass ceramics. Nevertheless, clinically favorable data were reported when feldspar ceramic restorations (Vita Mark II, Vita, Bad Säckingen, Germany) were adhesively luted using SBU combined with the composite cement RelyX Ultimate (3M Oral Care) without separate silanization; these data however represent only 18month short-term clinical effectiveness [22]. In this laboratory study, the solely YMPTS silanecontaining UA SBU applied without prior silanization not only underperformed $A D H-X T E$, but also the $\mathrm{RCP}+\mathrm{SB} 1-\mathrm{XT}$ control for each ceramic-surface pretreatment, this in terms of immediate as well as $3 \mathrm{~m}$ aged bond strength. No significant difference in bond strength with the RCP+SB1-XT control was recorded when SBU with prior silanization was bonded to AM-, TSC-, and HF-pretreated glass-ceramic surfaces. The inferior bonding performance of SBU, when glass ceramic was not beforehand separately silanized, has before been attributed to dehydration self-condensation of functional silanols within SBU due to YMPTS molecular instability in aqueous acidic solutions like UAs $[3,9,15,23]$. Nevertheless, without prior silanization ADH-XTE bonded equally effectively to TSC- and HF-prepared glass ceramic 
as the RCP+SB1-XT control (and thus more effectively than SBU), confirming the improved bonding potential of ADH-XTE without prior silanization to glass ceramic.

Although in-depth mechanistic research is needed to elucidate the reason(s) why the new experimental UA ADH-XTE performed significantly better than its precursor SBU, the following three hypothetical explanations have now been advanced. First, ADH-XTE makes use of special silane technology, combining the reactive silanol $(-\mathrm{Si}-\mathrm{OH})$ and amino groups $\left(-\mathrm{NH}_{2}\right)$ of APTES with polymerizable double carbon groups ( $\mathrm{C}=\mathrm{C}$ bonds) of $\mathrm{YMPTES}$. APTES is a bifunctional silane, enabling both its silanol and amino terminal groups to interact with hydroxyl groups present at the glass-ceramic surface [24]. Previous research demonstrated that APTES' silanol group preferentially adsorbed to zincoxide surfaces at low $\mathrm{pH}[25]$. Since ADH-XTE is acidic $(\mathrm{pH} \approx 3)$, its silanol group may have been the functional group that dominantly interacted with the glass-ceramic surface. While the amino functional group of APTES can make hydrogen bonds at the glass-ceramic surface, these amino groups may react with adjacent silanol groups to form aminosilanols and further cyclic internally hydrogen-bonded amine structures $[11,26,27]$. Hydrogen bonds between two adjacent amino groups may also be formed [26]. Such structures may all help stabilizing the hydrolyzed silanols at the ceramic surface. Hence, although the hydrogen bonds formed by the amino groups are not so stable as the siloxane -SiO-Si- bonds, they may still have contributed to bond strength. Furthermore, APTES has a propensity to form intermolecular siloxane crosslinks $[24,28]$. These crosslinks occur in regions of high siloxane density [29], which can make the silane interaction layer denser and contribute, in part, to withstand bond degradation. Second, YMPTES provides vinyl groups to covalently react with the resin component of the adhesive/cement. Note that the $-\mathrm{Si}-\mathrm{O}-\mathrm{C}_{2} \mathrm{H}_{5}$ group within YMPTES hydrolyzes relatively slowly compared with an $-\mathrm{Si}-\mathrm{O}-\mathrm{CH}_{3}$ group within other silanes, such as YMPTS [30]. This effect may have reduced potential dehydration self-condensation involving neighboring silanol groups. Third and finally, ADH-XTE may have outperformed SBU because of higher intrinsic physico-mechanical properties, such as flexural strength, elastic modulus and polymerization conversion. Eventually, the actual mechanism of interaction of the new experimental UA ADH-XTE and its contained silanes with glass ceramic needs to be fundamentally chemically analytically investigated using nuclear magnetic resonance (NMR) and/or Fourier transform infrared spectroscopy (FTIR). Such research may also disclose the best APTES/YMPTES ratio for optimum bonding efficacy and stability to HF-etched glass ceramic. 
Furthermore, while being promising without prior silanization, long-term clinical trials should confirm the applicability of the experimental adhesive ADH-XTE for simplified adhesive luting procedures of glass-ceramic restorations. Because the relatively low long-term hydrolytic stability of the silane might be a concern [31], shelf-life testing should be conducted to exclude gradual selfcondensation of silane with time within ADH-XTE, by which older solutions may gradually lose glassceramic bonding capacity.

\section{Conclusions}

The glass-ceramic bonding capacity of the new combined APTES/YMPTES silane-containing ADH-XTE surpassed that of its YMPTS silane-containing SBU precursor. HF etching still provides the best glassceramic bonding receptiveness, significantly better than TSC-prepared glass-ceramic surfaces. Solely chemical bonding of $\mathrm{ADH}-\mathrm{XTE}$ to $\mathrm{AM}$ - and MP-prepared glass-ceramic surfaces without micromechanical interlocking remains insufficient to achieve durable bonding to glass ceramics, irrespective with/without prior silanization. 


\section{References:}

[1] Tian T, Tsoi JK, Matinlinna JP, Burrow MF. Aspects of bonding between resin luting cements and glass ceramic materials. Dent Mater. 2014;30:e147-62.

[2] Politano G, Van Meerbeek B, Peumans M. Nonretentive bonded ceramic partial crowns: concept and simplified protocol for long-lasting dental restorations. J Adhes Dent. 2018;20:495-510.

[3] Yoshihara K, Nagaoka N, Sonoda A, Maruo Y, Makita Y, Okihara T, et al. Effectiveness and stability of silane coupling agent incorporated in 'universal' adhesives. Dent Mater. 2016;32:1218-25.

[4] Matinlinna JP, Lung CYK, Tsoi JK. Silane adhesion mechanism in dental applications and surface treatments: A review. Dent Mater. 2018;34:13-28.

[5] Söderholm KJM, Shang SW. Molecular-orientation of silane at the surface of colloidal silica. J Dent Res. 1993;72:1050-4.

[6] Antonucci JM, Dickens SH, Fowler BO, Xu HH, McDonough WG. Chemistry of silanes: interfaces in dental polymers and composites. J Res Natl Inst Stand Technol. 2005;110:541-58.

[7] Van Meerbeek B, Yoshihara K, Van Landuyt KL, Yoshida Y, Peumans M. From Buonocore's pioneering acid-etch technique to self-adhering restoratives. A status perspective of rapidly advancing dental adhesive technology. J Adhes Dent. 2020;22:7-34.

[8] Yao C, Zhou L, Yang H, Wang Y, Sun H, Guo J, et al. Effect of silane pretreatment on the immediate bonding of universal adhesives to computer-aided design/computer-aided manufacturing lithium disilicate glass ceramics. Eur J Oral Sci. 2017;125:173-80.

[9] Dimitriadi M, Panagiotopoulou A, Pelecanou M, Yannakopoulou K, Eliades G. Stability and reactivity of $\mathrm{Y}$-MPTMS silane in some commercial primer and adhesive formulations. Dent Mater. 2018;34:1089101.

[10] Pasternack RM, Rivillon Amy S, Chabal YJ. Attachment of 3-(aminopropyl)triethoxysilane on silicon oxide surfaces: dependence on solution temperature. Langmuir. 2008;24:12963-71.

[11] Xie Y, Hill CAS, Xiao Z, Militz H, Mai C. Silane coupling agents used for natural fiber/polymer composites: a review. Compos Part A Appl Sci Manuf. 2010;41:806-19.

[12] Gunda NSK, Singh M, Norman L, Kaur K, Mitra SK. Optimization and characterization of biomolecule immobilization on silicon substrates using (3-aminopropyl)triethoxysilane (APTES) and glutaraldehyde linker. Appl Surf Sci. 2014;305:522-30. 
[13] Van Landuyt KL, Snauwaert J, De Munck J, Peumans M, Yoshida Y, Poitevin A, et al. Systematic review of the chemical composition of contemporary dental adhesives. Biomaterials. 2007;28:3757-85. [14] Lung CY, Matinlinna JP. Aspects of silane coupling agents and surface conditioning in dentistry: an overview. Dent Mater. 2012;28:467-77.

[15] Yao C, Yu J, Wang Y, Tang C, Huang C. Acidic pH weakens the bonding effectiveness of silane contained in universal adhesives. Dent Mater. 2018;34:809-18.

[16] Qiao B, Wang TJ, Gao H, Jin Y. High density silanization of nano-silica particles using $\gamma^{-}$ aminopropyltriethoxysilane (APTES). Appl Surf Sci. 2015;351:646-54.

[17] Matinlinna JP, Vallittu PK. Bonding of resin composites to etchable ceramic surfaces - an insight review of the chemical aspects on surface conditioning. J Oral Rehabil. 2007;34:622-30.

[18] Rekow ED, Silva NR, Coelho PG, Zhang Y, Guess P, Thompson VP. Performance of dental ceramics: challenges for improvements. J Dent Res. 2011;90:937-52.

[19] Salvio LA, Correr-Sobrinho L, Consani S, Sinhoreti MA, De Goes MF, Knowles JC. Effect of water storage and surface treatments on the tensile bond strength of IPS Empress 2 ceramic. J Prosthodont. 2007;16:192-9.

[20] Peumans M, Valjakova EB, De Munck J, Mishevska CB, Van Meerbeek B. Bonding effectiveness of luting composites to different CAD/CAM materials. J Adhes Dent. 2016;18:289-302.

[21] Moro AFV, Ramos AB, Rocha GM, Perez CDR. Effect of prior silane application on the bond strength of a universal adhesive to a lithium disilicate ceramic. J Prosthet Dent. 2017;118:666-71.

[22] VogI V, Hiller KA, Buchalla W, Federlin M, Schmalz G. Controlled, prospective, randomized, clinical split-mouth evaluation of partial ceramic crowns luted with a new, universal adhesive system/resin cement: results after 18 months. Clin Oral Investig. 2016;20:2481-92.

[23] Chen B, Lu Z, Meng H, Chen Y, Yang L, Zhang H, et al. Effectiveness of pre-silanization in improving bond performance of universal adhesives or self-adhesive resin cements to silica-based ceramics: chemical and in vitro evidences. Dent Mater. 2019;35:543-53.

[24] Acres RG, Ellis AV, Alvino J, Lenahan CE, Khodakov DA, Metha GF, et al. Molecular structure of 3aminopropyltriethoxysilane layers formed on silanol-terminated silicon surfaces. J Phys Chem C. 2012;116:6289-97.

[25] Thomsen L, Watts B, Dastoor PC. A NEXAFS orientation study of $y$-aminopropyltriethoxysilane on 
zinc oxide surfaces. Surf Interface Anal. 2006;38:1139-45.

[26] Golub AA, Zubenko Al, Zhmud BV. Y-APTES modified silica gels: the structure of the surface layer. J Colloid Interf Sci. 1996;179:482-7.

[27] Park SJ, Jin JS, Lee JR. Influence of silane coupling agents on the surface energetics of glass fibers and mechanical interfacial properties of glass fiber-reinforced composites. J Adhes Sci Technol. 2000;14:1677-89.

[28] Howarter JA, Youngblood JP. Surface modification of polymers with 3-aminopropyltriethoxysilane as a general. Macromolecules. 2007;40:1128-32.

[29] Wang W, Vaughn MW. Morphology and amine accessibility of (3-aminopropyl)triethoxysilane films on glass surfaces. Scanning. 2008;30:65-77.

[30] Xiong M, Zhang K, Chen Y. ATRP of 3-(triethoxysilyl)propyl methacrylate and preparation of "stable" gelable block copolymers. Eur Polym J. 2008;44:3835-41.

[31] Zakir M, Ashrad U, Tian T, Han A, Qiao W, Jin XZ, et al. The role of silane coupling agents and universal primers in durable adhesion to dental restorative materials - a review. Curr Oral Health Rep. 2016;3:244-53. 
Table 1: Dental adhesives investigated in this study.

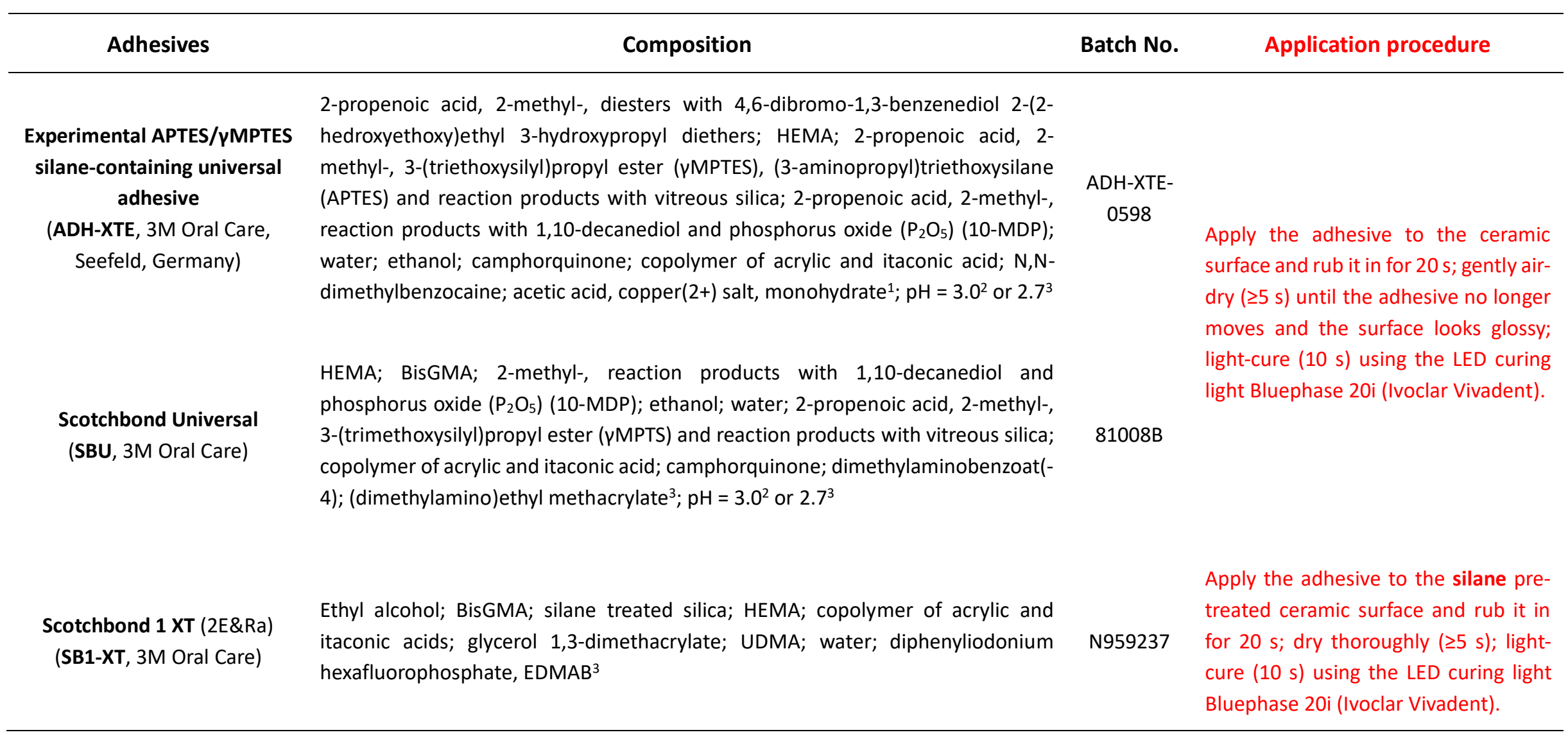

BisGMA: bisphenol A diglycidylmethacrylate; EDMAB: ethyl 4-dimethyl aminobenzoate; HEMA: 2-hydroxyethyl methacrylate; 10-MDP: methacryloxydecyl dihydrogen phosphate; UDMA: urethane dimethacrylate; ${ }^{1}$ According to $3 \mathrm{M}$ Oral Care Safety Data Sheet; ${ }^{2}$ Measured in-house using a digital $\mathrm{pH}$ meter (pH 3110, WissenschaftlichTechnische Werkstätten, Weilheim, Germany) equipped with the electrode MiniTrode (Hamilton Bonaduz, Bonaduz, Switzerland); ${ }^{3}$ According to $3 \mathrm{M}$ Safety Data Sheet. 


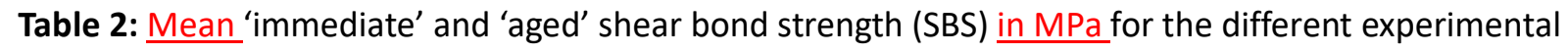
groups investigated.

\begin{tabular}{|c|c|c|c|c|}
\hline \multirow[t]{2}{*}{ Experimental groups } & \multicolumn{2}{|c|}{ Immediate SBS } & \multicolumn{2}{|c|}{ Aged SBS } \\
\hline & Mean $\left(\mathrm{SD}^{1}\right)$ & $\mathrm{ptf}^{2} / \mathrm{n}^{3}$ & Mean (SD) & $\mathrm{ptf} / \mathrm{n}$ \\
\hline As-milled (AM) + experimental ADH-XTE & $10.9(2.2)$ & $0 / 10$ & $0.1(0.3)$ & $8 / 10$ \\
\hline AM + RelyX Ceramic Primer (RCP; 3M Oral Care) & $22.3(3.5)$ & $0 / 10$ & $20.8(7.5)$ & $0 / 10$ \\
\hline \multicolumn{5}{|l|}{$+\mathrm{ADH}-\mathrm{XTE}$} \\
\hline AM + Scotchbond Universal (SBU, 3M Oral Care) & $4.8(1.1)$ & $0 / 10$ & $0.1(0.2)$ & $9 / 10$ \\
\hline$A M+R C P+S B U$ & $18.0(1.6)$ & $0 / 10$ & $14.2(6.1)$ & $0 / 10$ \\
\hline $\mathrm{AM}+\mathrm{RCP}+\mathrm{Scotch}$ bond $1 \mathrm{XT}$ (SB1-XT, 3M Oral Care) & $17.1(2.5)$ & $0 / 10$ & $13.3(2.7)$ & $0 / 10$ \\
\hline Tribochemically silica coated (TSC) + ADH-XTE & $27.2(2.1)$ & $0 / 10$ & $13.2(1.4)$ & $0 / 10$ \\
\hline $\mathrm{TSC}+\mathrm{RCP}+\mathrm{ADH}-\mathrm{XTE}$ & $33.1(2.3)$ & $0 / 10$ & $17.9(2.1)$ & $0 / 10$ \\
\hline $\mathrm{TSC}+\mathrm{SBU}$ & $14.4(2.1)$ & $0 / 10$ & $5.1(0.8)$ & $0 / 10$ \\
\hline $\mathrm{TSC}+\mathrm{RCP}+\mathrm{SBU}$ & $22.8(2.6)$ & $0 / 10$ & $14.2(2.3)$ & $0 / 10$ \\
\hline $\mathrm{TSC}+\mathrm{RCP}+\mathrm{SB} 1-\mathrm{XT}$ & $22.9(6.7)$ & $0 / 10$ & $11.1(2.0)$ & $0 / 10$ \\
\hline Hydrofluoric-acid etched (HF) + ADH-XTE & $30.2(5.6)$ & $0 / 10$ & $29.0(4.0)$ & $0 / 10$ \\
\hline $\mathrm{HF}+\mathrm{RCP}+\mathrm{ADH}-\mathrm{XTE}$ & $36.7(7.0)$ & $0 / 10$ & $36.0(3.5)$ & $0 / 10$ \\
\hline $\mathrm{HF}+\mathrm{SBU}$ & $22.6(3.7)$ & $0 / 10$ & $14.6(1.3)$ & $0 / 10$ \\
\hline $\mathrm{HF}+\mathrm{RCP}+\mathrm{SBU}$ & $32.5(2.9)$ & $0 / 10$ & $28.4(2.5)$ & $0 / 10$ \\
\hline$H F+R C P+S B 1-X T$ & $30.4(6.2)$ & $0 / 10$ & $29.9(4.3)$ & $0 / 10$ \\
\hline Mirror-polished (MP) + ADH-XTE & $8.7(2.8)$ & $0 / 10$ & $7.4(1.9)$ & $0 / 10$ \\
\hline $\mathrm{MP}+\mathrm{RCP}+\mathrm{ADH}-\mathrm{XTE}$ & $33.0(5.9)$ & $0 / 10$ & $26.4(5.0)$ & $0 / 10$ \\
\hline $\mathrm{MP}+\mathrm{SBU}$ & $5.0(0.7)$ & $0 / 10$ & $0.4(0.7)$ & $6 / 10$ \\
\hline $\mathrm{MP}+\mathrm{RCP}+\mathrm{SBU}$ & $20.4(5.1)$ & $0 / 10$ & $16.0(3.2)$ & $0 / 10$ \\
\hline $\mathrm{MP}+\mathrm{RCP}+\mathrm{SB} 1-\mathrm{XT}$ & $15.4(5.3)$ & $0 / 10$ & $11.0(5.2)$ & $0 / 10$ \\
\hline
\end{tabular}

${ }^{1} S D=$ Standard Deviation $;{ }^{2} p t f=$ pre-test failure $;{ }^{3} n=$ specimen number. 
Color version of the figures for pdf and online, black and white version for printing.

Figure legends

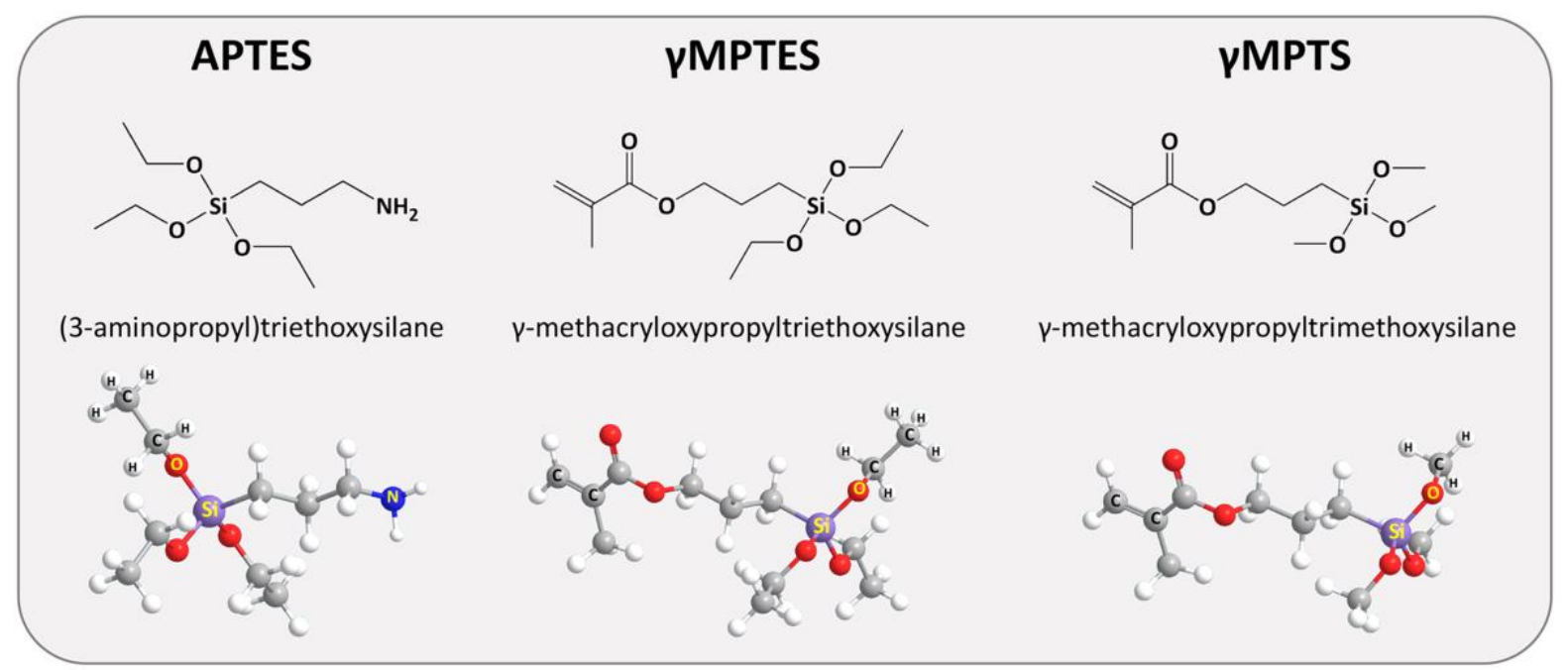

Fig. 1 - The molecular and 3D chemical structures of the three silane coupling agents investigated, namely APTES, $\gamma$ MPTES and $\gamma M P T S$.
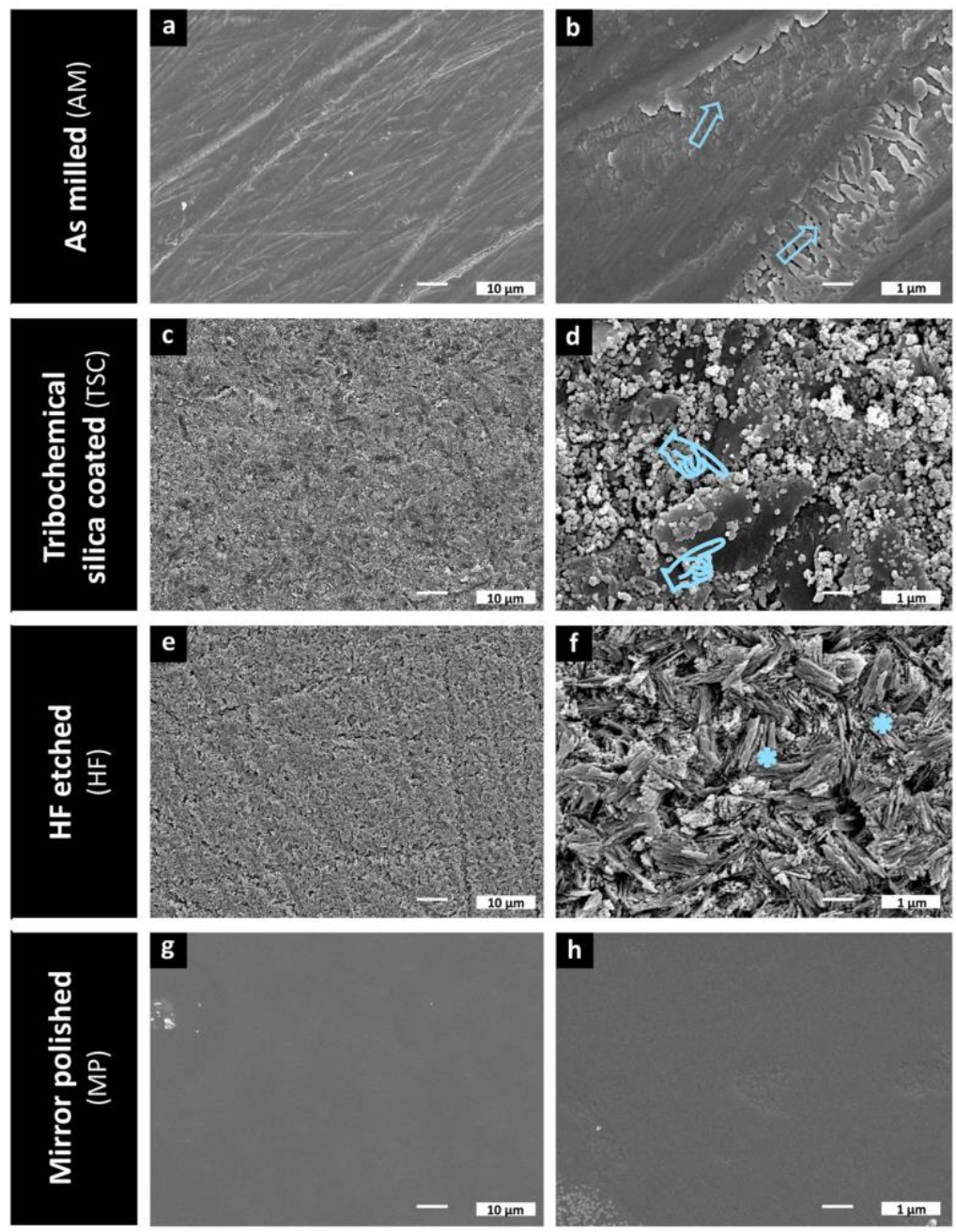
Fig. 2 - Representative SEM photomicrographs of as-milled (AM), tribochemical silica coated (TSC), hydrofluoric-acid etched (HF), and mirror-polished (MP) lithium-disilicate glass ceramic surfaces at low $1000 \times$ original magnification and high $10000 \times$ original magnification $\underline{\text { open light blue arrow: surface }}$ $\underline{\text { irregularities; light blue handpointers: silica; asterisks: needle-like crystals). }}$

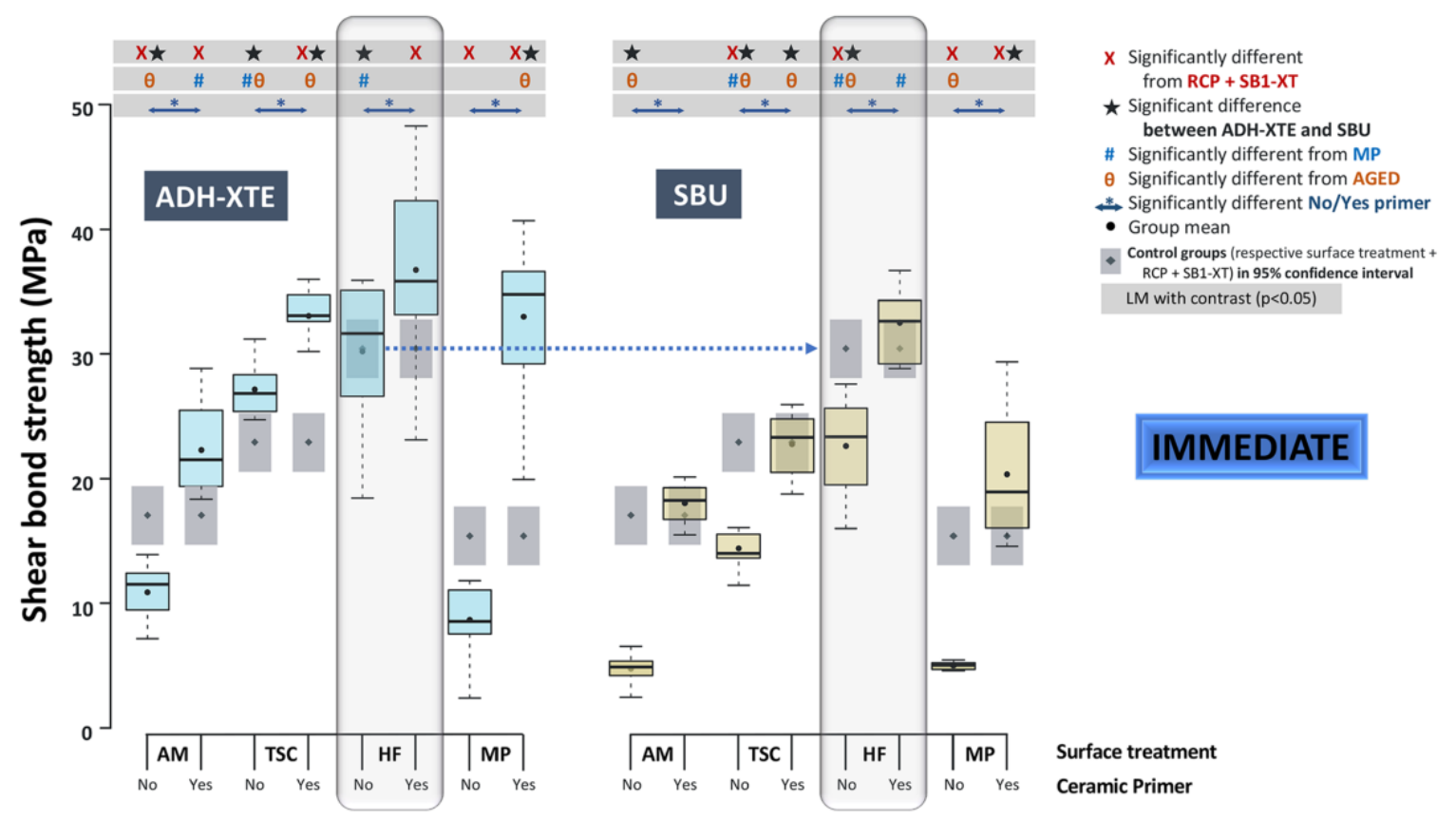

Fig. 3 - Box-and-whisker plots of immediate (1-week water storage) SBS (in MPa) of the experimental APTES/YMPTES silane-containing universal adhesive ADH-XTE and its commercial YMPTS silanecontaining pre-cursor Scotchbond Universal (SBU; 3M Oral Care) to AM-, TSC-, HF- and MP-prepared lithium-disilicate glass-ceramic surfaces without and with prior silanization using RelyX Ceramic Primer (RCP; 3M Oral Care). The box represents the data spreading between the first and third quartile, with the whiskers denoting minimum and maximum. Black closed dots represent mean SBS with the horizontal line referring to the median. Grey boxes in the background represent the $95 \%$ confidence interval of the control consisting of prior silanization with RCP followed by the application of the twostep etch-and-rinse adhesive Scotchbond 1 XT (3M Oral Care). Statistically significant differences $(p<0.05)$ are indicated by specific symbols in the figure legend. 


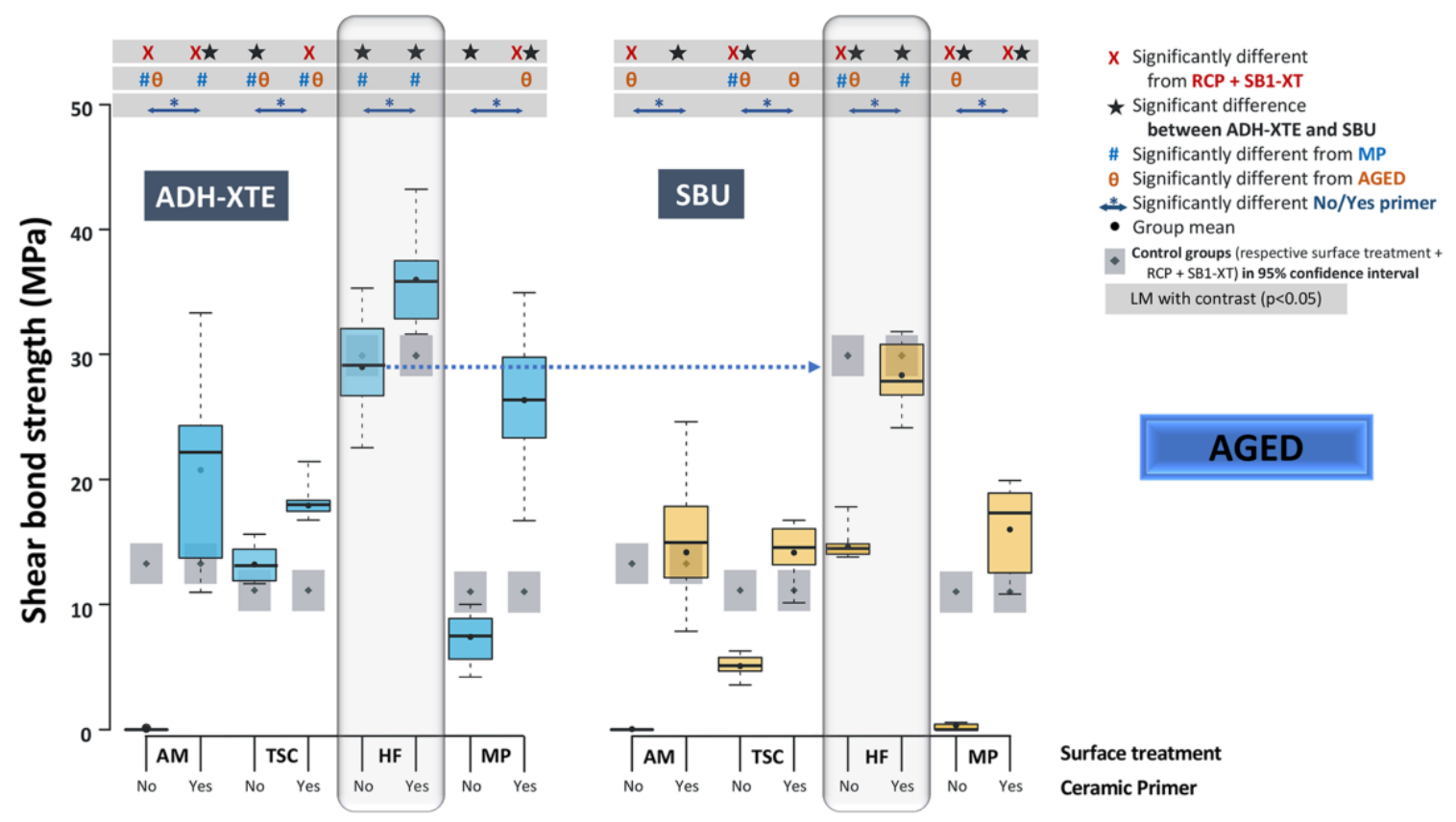

Fig. 4 - Box-and-whisker plots of aged (3-month water storage) SBS (in MPa) of the experimental APTES/YMPTES silane-containing universal adhesive ADH-XTE and its commercial YMPTS silanecontaining pre-cursor Scotchbond Universal (SBU; 3M Oral Care) to AM-, TSC-, HF- and MP-prepared lithium-disilicate glass-ceramic surfaces without and with prior silanization using RelyX Ceramic Primer (RCP; 3M Oral Care). The box represents the data spreading between the first and third quartile, with the whiskers denoting minimum and maximum. Black closed dots represent mean SBS with the horizontal line referring to the median. Grey boxes in the background represent the $95 \%$ confidence interval of the control consisting of prior silanization with RCP followed by the application of the twostep etch-and-rinse adhesive Scotchbond 1 XT (3M Oral Care). Statistically significant differences $(p<0.05)$ are indicated by specific symbols in the figure legend. 

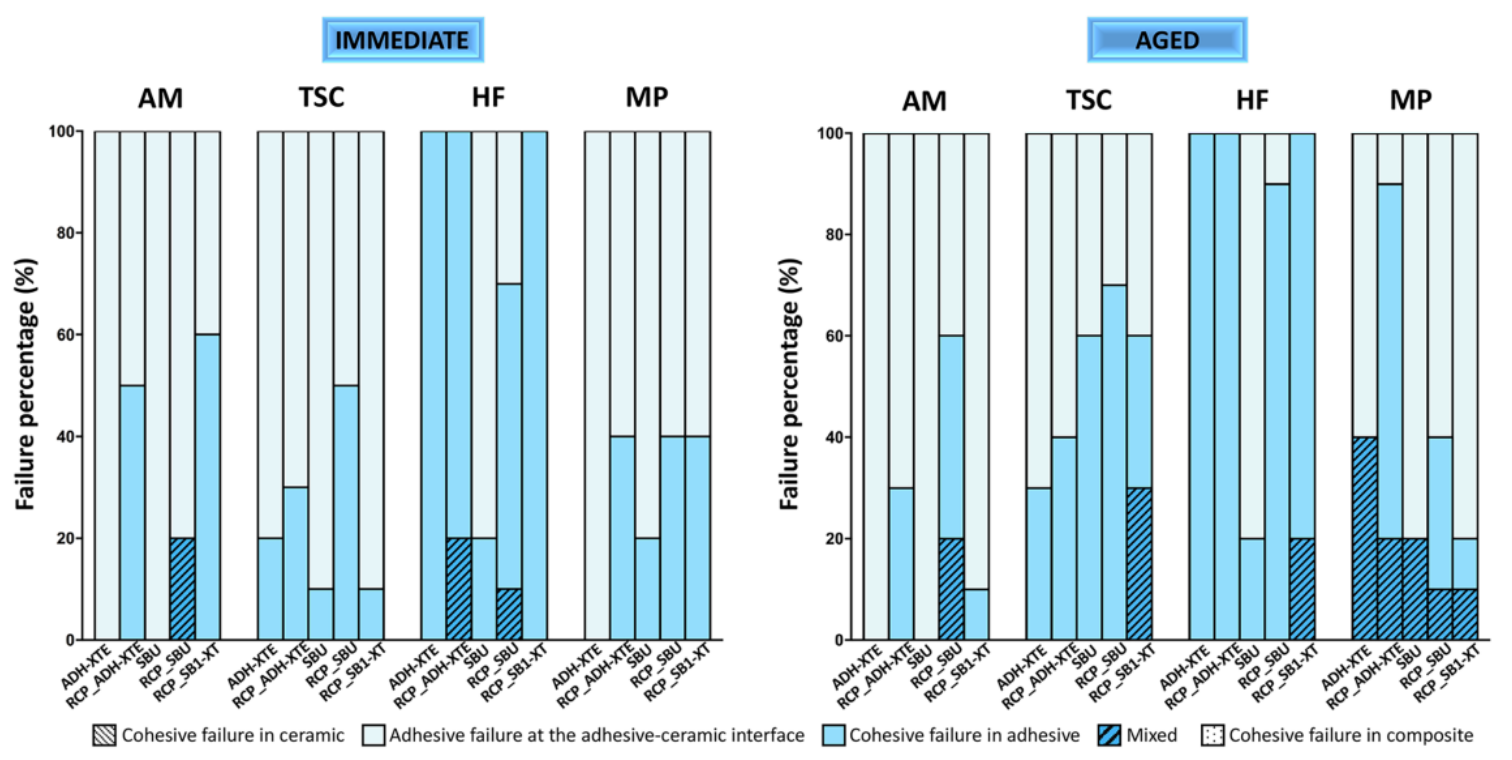

Fig. 5 - Immediate and aged SBS failure modes recorded for the different experimental groups. When bonded to HF-etched glass-ceramic surfaces, the specimens failed predominantly cohesively in the adhesive, except when SBU was applied without separate prior silanization. The primary failure mode of all other experimental groups was 'adhesive failure at the adhesive-ceramic interface'.

A

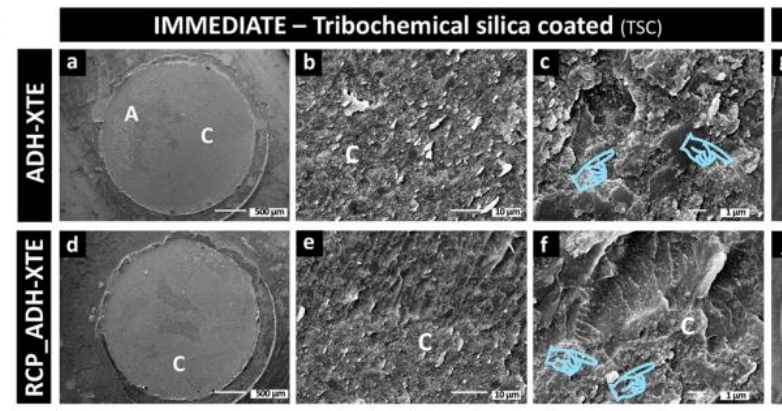

B

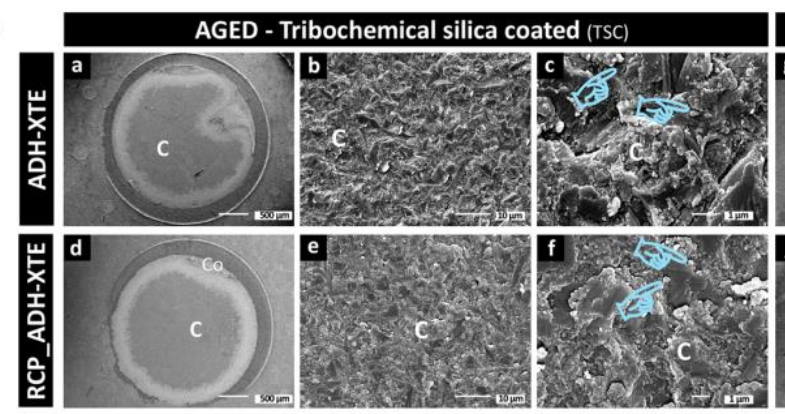

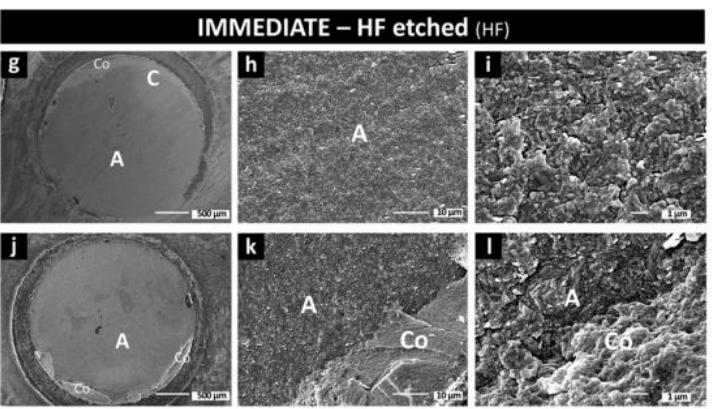
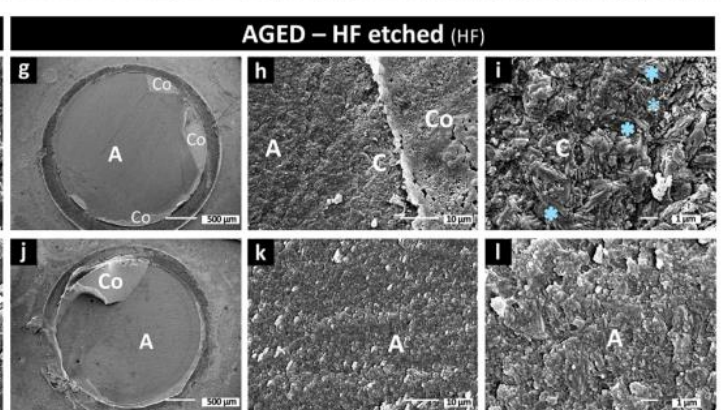

Fig. 6 - Representative SEM photomicrographs of immediate (A) and aged (B) fractured surfaces of SBS specimens subjected to tribochemical silica coated (TSC) glass-ceramic surfaces in (a-f) and HF-etched (HF) glass-ceramic surfaces in ( $\mathrm{g}-\mathrm{l})$, of which the fractured glass-ceramic side is presented. Regarding immediate fractured TSC specimens in (A), ADH-XTE revealed an adhesive failure at the adhesive- 
ceramic interface with adhesive fragments that remained attached to the ceramic surface, photographed at 35x original magnification in (a) with higher magnification in (b) and (c). In (d), ADHXTE applied with prior silanization (RCP_ADH-XTE) revealed an adhesive failure at the adhesive-ceramic interface, photographed at $35 \times$ original magnification with higher magnification in (e) and (f). Regarding immediate fractured HF specimens in (g), ADH-XTE revealed a cohesive failure in adhesive resin, photographed with higher magnification in (h) and (i). In (j), RCP_ADH-XTE revealed a mainly cohesive failure in adhesive resin with composite fragments remaining attached onto the fractured surface, photographed with higher magnification in $(k)$ and $(I)$. Regarding aged fractured TSC specimens in (B), ADH-XTE in (a-c) and RCP_ADH-XTE in (d-f) present with an adhesive failure at the adhesiveceramic interface. Regarding aged fractured HF specimens, ADH-XTE in (g-i) and RCP_ADH-XTE in (j-I) revealed a cohesive failure in adhesive resin with composite fragments remaining attached onto the fractured surface. Upon aging, the adhesive layer was still complete and uniform for RCP_ADH-XTE. A: adhesive; C: ceramic; Co: composite. Light blue handpointers: silica; asterisks: needle-like crystals.

\section{Graphical abstract}

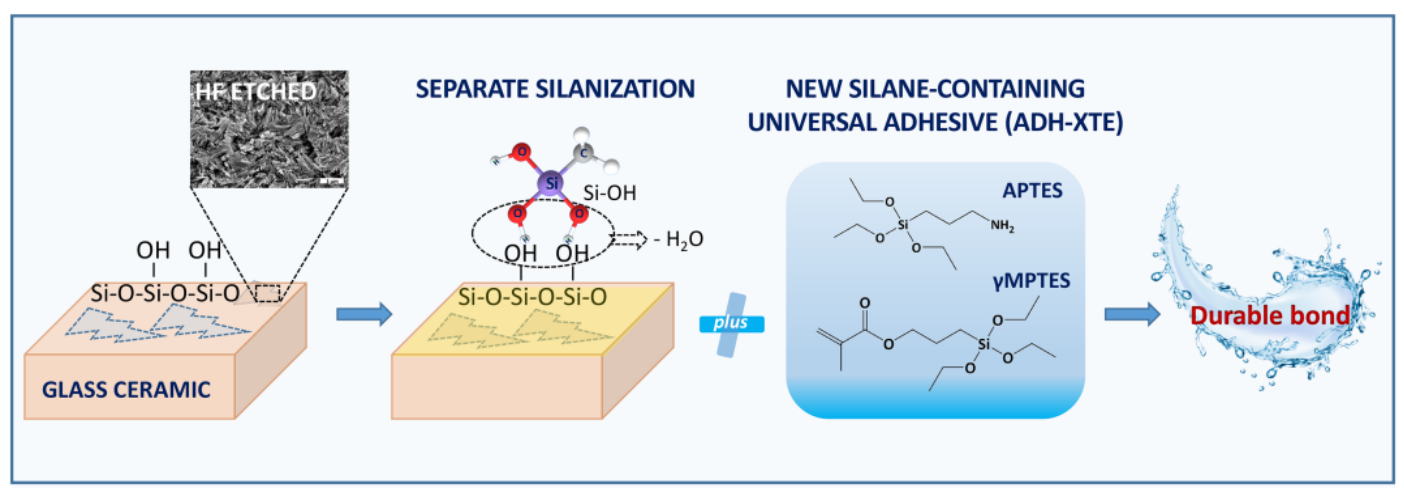

